\title{
It Is Necessary to Abrogate All the International Basic Standards of Seals
}

\author{
Xu Changxiang, Zhang Xiaozhong, Chen Youjun
}

Baoyi Group Co. Ltd., Wenzhou, Zhejiang, 325105, China

Email address:

xchx9763@sina.com (Xu Changxiang), zxz@baoyi.com (Zhang Xiaozhong),info@baoyi.com (Chen Youjun)

\section{To cite this article:}

Xu Changxiang, Zhang Xiaozhong, Chen Youjun. It Is Necessary to Abrogate All the International Basic Standards of Seals. Special Issue: Xu's Sealing and Flowing Theories of Fluids. International Journal of Energy and Power Engineering. Vol. 5, No. 4-1, 2016, pp. 1-12. doi: 10.11648/j.ijepe.s.2016050401.11

Received: June 22, 2016; Accepted: June 25, 2016; Published: August 24, 2016

\begin{abstract}
According to definitions in the international standard JCGM 200, physical quantities, divided into base quantity and derived quantity, are a property of a phenomenon, body, or substance, where the property has a magnitude that can be expressed as a number and a reference (a measurement unit); a base quantity cannot be expressed in terms of the others, and a derived quantity can be defined in terms of the base quantities of its system; and these base and derived quantities are related to each other by some laws and their equations. In the International System of Quantities, tightness or leak resistance is a derived quantity defined by a sealing or leaking law, which is the product of pressure $p$ and time $t$ expended on leaking a unit cubage of fluid through the sealing joints of a pressure vessel or system at a constant pressure, but by no means the reciprocal of "leakage rate" fabricated by the international standards. Various fluid circuits in pipes, on objects and in joints differ only in their fluid (leak) resistance and reactance, and it is impossible to know how to identify and control a fluid circuit before knowing these physical quantities. All the international basic standards of seals should be abrogated as soon as possible because they were so enacted before knowing what the leak resistance (tightness) and the qualified seal are and how to design, install and inspect a seal that it is impossible to count on them providing any effective control of leakage for a pressure vessel or system.
\end{abstract}

Keywords: physical quantity, fluid resistance, leak resistance (tightness), fluid reactance, seal, fluid circuit

\section{Introduction}

Teachers, in their rudimentary teaching of electricity, always explain to students an invisible electric flow in wires under an invisible voltage by analogy with fluid flow in pipes under a pressure ${ }^{[1-2]}$.

On the other hand, engineers, in their research of fluid flow, always reason out laws of fluid flow according to known laws of electric flow.

Poiseuille, a French physicist and physiologist, in 1938, and Hagen, a German engineer, in 1939, separately proved by experiments that the relation between the fluid resistance $R_{F}$ of a pipe and the fluid current $I_{F}$ through the pipe under a pressure $p$ between its two ends $\left[R_{F}=p / I_{F}=(8 \eta l) /\left(\pi r^{4}\right)\right.$, where $\eta=$ fluid viscosity, $l=$ pipe length, $r=$ pipe wall radius] substantially corresponds thoroughly to the relation between the electric resistance $R$ of a wire and the electric current $I$ through the wire under a voltage $U$ between its two ends $[R=$
$U / I=(\rho l) / A$, where $\rho=$ electrical resistivity, $l=$ wire length, $A$ $=$ wire cross-sectional area $]^{[3]}$.

Chinese engineers recently have discover and proved:

- that the relation between the fluid reactance $X_{F}$ of a fluid circuit and the fluid current $I_{F}$ through the circuit under a pressure $p$ between its two ends $\left[X_{F}=p / I_{F}=(\rho v) /(2 A)\right.$, where $\rho=$ fluid density, $v=$ fluid velocity, $A=$ fluid cross-sectional area] substantially corresponds thoroughly to the relation between the electric reactance $X$ of an electrical circuit and the electric current $I$ through the circuit under a voltage $U$ between its two ends $[X=U / I=$ $2 \pi f L$ or $1 /(2 \pi f C)$, where $f=$ electric frequency, $L=$ electric inductance, $C=$ electric capacitance],

- that the relation between the leak resistance $R_{L}$ (tightness) from a fluid vessel to the atmosphere and the leakage current $I_{L}$ through the leak circuit of the vessel under a pressure $p\left[R_{L}=p / I_{L}=p t / C\right.$, where $C=$ cubage of fluid leaked for a duration $t$ of a constant pressure $p$ ] substan- 
tially corresponds thoroughly to the relation between the leak resistance $R$ (insulativity) from an electric capacitor to the earth and the leakage current $I$ through the leak circuit of the capacitor under a voltage $U[R=U / I]$, and

- that various electrical circuits differ only in their electric (leak) resistance and reactance and various fluid circuits in pipes, on objects and in joints differ only in their fluid (leak) resistance and reactance ${ }^{[4]}$. For example, the difference between a very thin tube circuit and a general pipe circuit is only that the former can only have a fluid velocity so low as to be regarded as one only with fluid resistance but no fluid reactance, and the latter also has a fluid velocity so considerable as to cause it to have a considerable fluid reactance and a considerable pressure drop. People have wrongly thought that Poiseulle's law is applicable only to a very thin tube circuit but not to a general pipe circuit before not discovering the physical quantity of fluid reactance ${ }^{[5]}$.

It is imaginable that it is obviously impossible to know how to identify and control a fluid flow without physical quantities of fluid resistance and reactance, and that it is also obviously impossible to know how to identify and control a fluid leakage without a physical quantity of fluid leak resistance. Therefore, it is very necessary to see what mistakes and ignorance there are in the present international basic standards of seals that closely relate to man's life and property safety after reasoning out the physical quantities of fluid resistance, leak resistance and reactance according to the physical quantities of electric resistance, leak resistance and reactance.

\section{The Present Standards Have Mistaken the Reciprocal of Leakage Current for Tightness, Which Is Equivalent to Not Knowing What Tightness Is}

As far as flow is concerned, electricity is one fluid. Of course, it can be known that fluid leak resistance is fluid tightness according to the definitions of electrical insulativity and leak resistance, and that cubage of fluid leaking through a sealing joint for a unit of time should be called fluid leakage current according to the definition of electric (leakage) current. Accordingly, it is undoubtedly incorrect for the present standards to call the "volume of fluid leaking to the atmosphere through a sealing joint for a unit of time" the "leakage rate" [6 10].

A pressure vessel or system with a smaller leakage current at a fixed pressure is one with a better tightness, and vice versa. Due to that, the present standards identify tightness by regarding "leakage current" as "leakage rate", which is equivalent to considering the reciprocal of "leakage current" to be the "tightness" [11].

As tightness or leak resistance $R_{L}, R_{L}=p / I_{L}$, is a constant not changing with pressure $p$ and leakage current $I_{L}$, and leakage current $I_{L}, I_{L}=p / R_{L}$, a variable changing with pressure $p$, it is equivalent to using a variable quantity of "leakage current" to identify a constant quantity of "tightness" and so it cannot identify any real goodness or badness of a seal for the present standards to regard the reciprocal of a leakage current under an uncertain fixed pressure as the tightness.

Because tightness or leak resistance $R_{L}=p / I_{L}=p t / C$, where $C=$ cubage of fluid leaked for a duration $t$ of a constant pressure $p$, it is equivalent to using the "fluid volume decompressed in uncertain proportion" to identify the "compressed fluid volume" and it causes the test result to be far away from its truth value and to have no worthiness for the present standards to identify tightness by regarding the "volume of fluid leaking to the atmosphere through a sealing joint for a unit of time" as the "leakage rate" [8 10].

In fact, fluid volatility and adhesivity cause fluid leaking at a lowest velocity not to collect and gauge accurately at all. Accordingly, the present standards have had to use counting of "number of liquid drops or gas bubbles leaking to the atmosphere for a unit of time" to replace gauging of "the volume of fluid leaking to the atmosphere for a unit of time". The inspection of "no visually detectable leakage" relies on macroscopic continuous observation under a fixed test pressure, and any leakage will cause a test pressure decay and a decrease of leakage, so that it is impossible to identify the goodness or badness of a seal by leakage current under a fixed test pressure; or the leakage-detecting method specified by the present standards cannot identify and control the quality of seals at all on the basis of "volume of fluid leaking to the atmosphere for a unit of time".

Therefore, from their mistaking the reciprocal of leakage rate that should be called leakage current for tightness, it can be known that the present standards have not still known what tightness is.

\section{The Present Standards Have Not Known What a Circumferential Uniform Mo- mentary Leakage Is, Which Is Equivalent to Not Knowing What a Qualified Seal Is}

Because the leakage of a qualified seal will happen only when its contact-tightening stress is slightly smaller than the fluid pressure and any leakage of it will successively result in a fluid pressure decay, a rise of its sealing stress and the termination of the leakage, any leakage of a seal that is circumferentially fully uniformly tightened will happen only in a way of circumferential uniform momentary leakage at the moment when its sealing stress is counteracted at the same time by leaking pressure to approximate null in each orientation, or at the moment when its total pre-tightened force is slightly smaller than the sum of its unloading and unsealing actuation forces, or else it is not uniformly tightened circumferentially. If leakage is allowed not to happen in a way of circumferential uniformity, there will be no parameter of maximum unsealing actuation force that equals the product of fluid pressure and sealing contact area and is needed to calculate the seal's minimum total fastener load, or there will be no necessary condition for designing an effective seal. Hence, it can be said that any seal can only be designed according to a circumferential uniform momentary leakage or according to no local long 
leakage by dropping or bubbling. It is equivalent to inspecting seals according to "local long leakage" for the present standards to inspect seals according to "number of liquid drops or gas bubbles leaking to the atmosphere for a unit of time" ${ }^{[8-10] \text {, }}$ which fully proves that the present standards have not known the significance of a circumferential uniform momentary leakage and a local long leakage by dropping or bubbling to a qualified seal, or have not known what a qualified seal is.

\section{The Present Standards Have Not Known What a Sealing Mechanism Is, Which Is Equivalent to Not Knowing How to Design a Cavity of Self-Energizing Seals ${ }^{[12]}$}

The essence of self-energizing seals is causing a sealing ring, for example, a face sealing ring 02 in Fig.1, to exactly orthogonally transmit a fluid pressure or to exactly convert the fluid pressure $p$ on its internal cylinder into the sealing stress $S$ on its end face, and hence it is the material with a full liquid behavior that can be simply used for self-energizing rings.

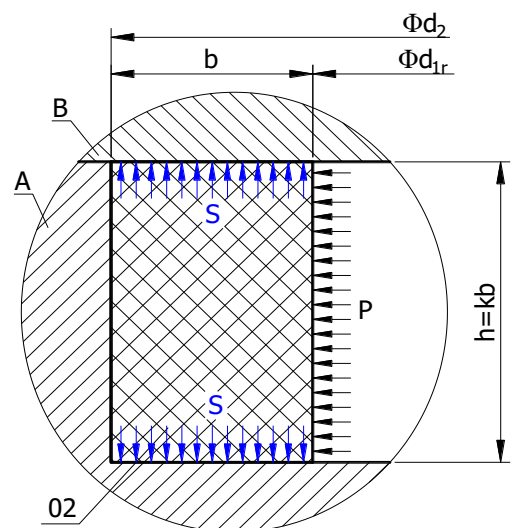

A Designed (port) end B Fully flat (cover) end 02 Self-energizing seal

Fig.1 Essence of self-energizing seals

The property that a liquid can transmit a pressure equally in each direction originates from its volume incompressibility during flow and deformation under a pressure. It can be seen from bulk modulus $K=E /[3(1-2 v)]$ that an object whose Poisson's ratio $v$ is closer to 0.5 has a volume incompressibility closer to infinity. The Poisson's ratio of a general object under normal temperature is greater than zero and smaller than 0.5 , but will be close to 0.5 when its homologous temperature, which is the ratio of its absolute temperature to its melting absolute temperature, is higher than 0.5 , and the closer to 1 (the melting point) its homologous temperature, the closer to 0.5 its Poisson's ratio, and vice versa. Thus it can be said that the Poisson's ratio is an index of liquid behavior and volume incompressibility of a general object, and that the closer to 0.5 its Poisson's ratio, the fuller its liquid behavior. Therefore, any material that has a Poisson's ratio close to 0.5 and can be deformed under a fluid pressure, such as rubber, PTFE, lead, gold etc., can be simply used for self-energizing rings.

Now that the essence of a self-energizing seal is causing a sealing ring to exactly orthogonally transmit a fluid pressure, and determined by whether its Poisson's ratio or orthogonal strain ratio of its material can be up to 0.5 or not under a fluid pressure, thus any pliable solid material, no matter how smaller than 0.5 its Poisson's ratio is, can be used for a self-energizing ring by compensating for its orthogonal strain ratio up to 0.5 by an angle $\theta_{l}$ shown in Fig.2.

As shown in Fig.3, any radial clearance $(C)$ between the

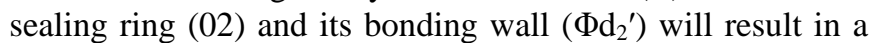
Poisson's deformation making it increased in circumference and decreased in height at a certain pressure, and cause it to be away from its previous sealed contact. In fact, it is without both radial and axial clearances at the same time that a sealing ring with a full liquid behavior can exactly orthogonally transmit a fluid pressure or can be self-energized to its leak-free state in time relying on its incompressibility. Because both ring's manufacture error and material's thermal expansion coefficient difference will cause a sealing ring a possible radial contact clearance, any self-energizing ring with full liquid behaviors still needs an angle $\theta_{c}$ compensating for its radial contact to be able to in time offset its orthogonal deformation proportional to its Poisson's ratio value and caused by its possible radial clearance under pressures.

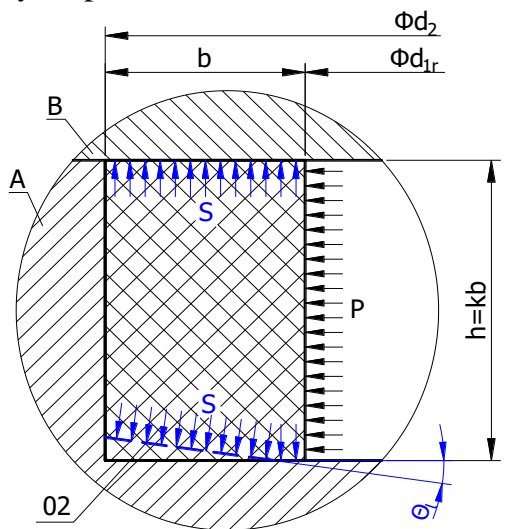

A Designed (port) end B Fully flat (cover) end 02 Self-energizing seal

Fig.2 Compensation for orthogonal deformation of sealing rings

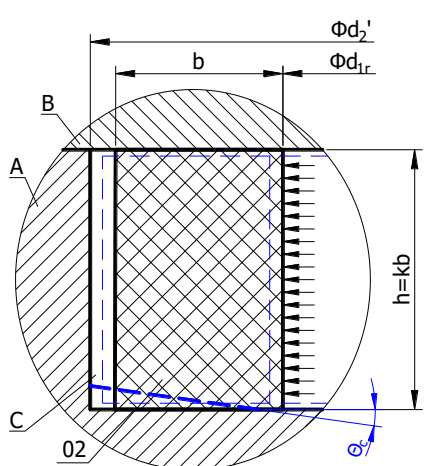

A Designed (port) end B Fully flat (cover) end 02 Self-energizing seal

Fig.3 Offset to orthogonal deformation of sealing rings

The compensation of a sealing ring for its Poisson's orthogonal deformation or its liquid behavior is aimed at compensating for its insufficient increment in height caused by a less than 0.5 Poisson's ratio when its radial contact has no 
clearance, and the compensation for its radial contact, aimed at offsetting its decrease in height that is proportional to its Poisson's ratio value and caused by its possible radial clearance; i.e. it is necessary for a self-energizing ring to be compensated for its liquid behavior and for its radial contact, and both are to increase its deformation in height under a fluid pressure. Because a general sealing material has a Poisson's ratio ranging from 0 to 0.5 , any self-energizing ring will need or have one angle $\theta_{l}$ fully compensating for its liquid behavior and one angle $\theta_{c}$ fully compensating for its contact whose magnitudes are both determined by the Poisson's ratio limit $(0.5)$ if the compensation for its liquid behavior is done from 0 to 0.5 and the compensation for its contact is done from 0.5 to 0 . The two full compensation angles can be unifiedly called an essential Poisson's deformation compensation or offset angle $\theta_{e}=\operatorname{arctg}(v h) / r=h / d$, where $v=$ Poisson's ratio limit $=0.5, h=$ height of self-energizing rings, $d=2 r=$ inner diameter of self-energizing rings.

A self-energizing ring is designed to deform under any pressure. The wedging function of its essential Poisson's deformation compensation or offset angle $\theta_{e}$ can only cause it to have some useful sealing deformation. However great the angle $\theta_{e}$ is, what it changes is only the time for the ring material to reach its Poisson's ratio limit 0.5 or 0 but never the magnitude of the two limits, or at most compensates the ring material for its orthogonal deformation ratio from 0 to 0.5 or offsets its orthogonal deformation ratio from 0.5 to 0 as soon as possible, or at most eliminates the lagging of its orthogonal deformation ratio behind its final value. Therefore, any material of self-energizing rings, however great its Poisson's ratio is, can use one angle $\theta_{x}$ greater than $\theta_{e}$ as its Poisson's deformation compensation angle $\theta_{l}$ and offset angle $\theta_{c}$ when the influence from its thermal expansion coefficient difference can be ignored.

The essence of the necessary essential Poisson's deformation compensation or offset angle $\theta_{e}$ of a self-energizing ring is to ensure that the ring cavity can cause the ring a self-energizing deformation from a great space to a small space under a fluid pressure. Besides, the ratio $k$ of height $h$ to wall thickness $b$ of self-energizing rings shall be great enough to cause ring's inner cylinder $\left(\phi d_{l r}\right)$ area to be greater than ring's end face area in order to ensure that ring's self-energizing actuation force is greater than ring's unsealing actuation force. The present standards of seals were enacted before discovering the sealing mechanism of self-energizing rings, and may not have any correct seal-designing requirement for a qualified sealing cavity.

\section{The Present Standards Have Not Known the Sealing Difficulty Factor $m_{1}$, Which Is Equivalent to Not Knowing How to Design a Sealing Element ${ }^{[12]}$}

A fully leak-free connection results from loading a sealing contact layer first up to its fully deformed contact and then up to its fully tight contact, or needs first to create a deformed contact that can fully seat a sealing surface into ir- regularities on a surface to be sealed and then to create a tight contact that can fully resist a uniform leakage. Because the active elastic modulus of a compressed contact layer or surface is a seal's capability parameter to resist a sealing deformation and a tightening contact and the active elastic modulus of the substrate of a compressed contact layer or surface is a seal's power parameter to achieve and maintain the sealing deformation and the tightening contact, the closer to 1 the material's elastic modulus ratio of the compressed contact layer to the substrate of a sealing element, the more difficult to be assembled to a fully deformed contact the sealing element; the closer to 1 the active elastic modulus ratio of the contact surface to the substrate of a sealing element that has been up to a fully tight contact, the more difficult to maintain the realized tight contact the sealing element; and vice versa. In other words, the difficulty for a sealing element to achieve or maintain its fully leak-free connection is determined by material's or active elastic modulus ratio $E_{d} / E_{s}$ of its contact layer or surface to its substrate, and the closer to 1 the value of $m_{l}, m_{l}=E_{c} / E_{s}$, of a sealing element, the more difficult to achieve or maintain a fully leak-free connection the sealing element. Therefore, a hard sealing element coated with grease, whose $E_{c} \equiv 0$ or $m_{l} \equiv 0$, is the easiest to achieve or maintain a fully leak-free connection; a sealing element made of coreless rubber (elastomer), whose $E_{c} \equiv E_{s}$ or $m_{1} \equiv 1$ because it has no yield point, is the most difficult to achieve or maintain a fully leak-free connection; and the other metallic sealing element that can result in $E_{c} \ll E_{s}$ or $m_{l} \ll 1$ by designing a weak contact layer or a soft coating and a strong substrate and by being assembled separately to a fully (plastically) deformed contact and a fully elastically deformed support is far easier to achieve or maintain a fully leak-free connection than the coreless rubber element (see Fig.4). However, the present standards, which measure the sealing difficulty or easiness of sealing elements only according to their material strength, have wrongly thought what is the easiest to achieve or maintain a leak-free connection is coreless rubber sealing elements and what is the most difficult to achieve or maintain a leak-free connection is metallic sealing elements ${ }^{[13]}$.

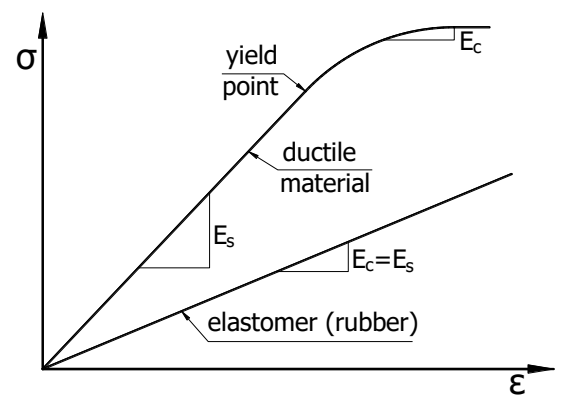

Fig.4Xu's sealing difficulty factor $m_{1}=E_{c} / E_{s}$

It can be seen from the concept of Xu's sealing difficulty factor that a sealing element shall be designed to have a soft and inelastic contact layer and a strong and elastic contact layer substrate, and also ensure that its contact surface and its substrate can be assembled or self-energized separately to a 
full (plastic) deformation and a full elastic deformation. The present standards of seals were enacted before discovering the sealing difficulty factor, and may not have any correct seal-designing requirement for a qualified sealing element.

\section{The Present Standards Have Mistaken the Seal-Working or Installing Stress in Position for the Sealing Stress, Causing No Seal to Have Correct Fastener Load and Installation}

The minimum necessary sealing stress $y$ is the minimum compressive stress required for a qualified sealing element to maintain a fully leak-free contact at atmospheric temperature and pressure.

Any qualified seal needs a sealing surface that has microcosmic irregularities fully complementary to the surface to be sealed and that can be qualified only by installing or compressively working it in position. Accordingly, the minimum necessary sealing stress $y$ is not the compressive stress at the moment when a sealing element at atmospheric temperature and pressure is installed or loaded up to its no visually detectable leakage, but shall be the compressive stress on the contact surface at the moment just before the sealing contact is circumferentially uniformly unloaded up to its uniform leakage in the original position where it was circumferentially uniformly loaded up to its fully leak-free state. In other words, the minimum necessary sealing stress $y$ includes a deforming load component $\left(y_{e}\right)$ required to deform the qualified sealing element with an elastic modulus remnant $E_{c}$ in its contact surface (see Fig.4) up to a fully deformed contact and a tightening load component $\left(y_{a}\right)$ required to tighten the fully deformed contact up to a fully tight contact that can fully resist atmospheric pressure (0.1 MPa), or $y=y_{e}+y_{a}$ and $y_{a}=0.1 \mathrm{MPa}$.

From the fact that grease can be easily deformed and not return to its original shape at atmospheric pressure, it can be seen that the deforming load component $y_{e}$ of a hard sealing element coated with grease is null or $y_{e}=0$. From the fact that rubber can be easily deformed and return to its original shape at atmospheric pressure, it can be seen that the deforming load component $y_{e}$ of a coreless rubber O-ring is $0.1 \mathrm{MPa}$ or $y_{e}=$ 0.1 . Because a hard sealing element coated with grease and a coreless rubber sealing element (elastomer) are separately the easiest and the most difficult to achieve or maintain a fully leak-free connection, all the qualified sealing elements have a lower limit of minimum necessary sealing stresses $y_{\min }=0.1$ MPa (equal to the $y$ 's value of hard sealing elements coated with grease) and an upper limit of minimum necessary sealing stresses $y_{\max }=0.2 \mathrm{MPa}$ (equal to the $y$ 's value of coreless rubber O-rings); or any sealing element is unqualified whose minimum necessary sealing stress $y$ is more than $0.2 \mathrm{MPa}$.

The leak-free maintenance factor $m_{2}$ is the ratio of sealing actuation force $F_{s}$ to maximum unsealing actuation force $F_{u}$ of a qualified seal, or $m_{2}=F_{s} / F_{u}$. Because the force $F_{u}=$ the leaking-fluid's unsealing actuation area or the sealing contact area $A_{u} \mathrm{x}$ the fluid pressure $p$, the force $F_{u}$ will equal zero when the seal is both in a leak-free state (because the pressure $p$ on the contact surface equals zero) and in a locally leaky state (because the leaking-fluid's actuation area approximates to zero), or will emerge only at the moment when a circumferential uniform leakage happens caused by a null sealing stress at the moment when the force $F_{u}=$ the force $F_{s}$, and also disappear with a leakage-caused pressure decay. Therefore, any qualified seal has a leak-free maintenance factor $m_{2}$ equal to 1 at the uniform leakage moment, and a leak-free maintenance factor $m_{2}$ equal to $\infty$ during the leak-free state and the non-uniform leakage state.

From the definitions of the minimum necessary sealing stress $y$ and the leak-free maintenance factor $m_{2}, m_{2}=F_{s} / F_{u}=$ $\left(F_{\Sigma}-F_{i}\right) / F_{u}$, for a qualified seal, it can be known:

- that the condition for a qualified pressure-tight seal to maintain its leak-free state is:

$\left(F_{s}-F_{u}\right) \geq y A_{u} \rightarrow\left(m_{2} p A_{u}-p A_{u}\right) \geq y A_{u} \rightarrow m_{2} \geq(1+y / p)$; and

- the condition for a qualified self-energizing seal to maintain its leak-free state is:

$\left(F_{s}-F_{u}\right) \geq y A_{u} \rightarrow\left(p A_{s}-p A_{u}\right) \geq y A_{u} \rightarrow m_{2}=A_{s} / A_{u} \geq(1+y / p) ;$ where $F_{s}=$ sealing actuation force, $F_{u}=$ maximum unsealing actuation force, $F_{\Sigma}=$ minimum total fastener load, $F_{i}=$ fluid unloading actuation force (in sealing contact minor circle), $y$ $=$ minimum necessary sealing stress, $p=$ fluid pressure, $A_{s}=$ fluid sealing actuation area, and $A_{u}=$ sealing contact area. Hence, it can be seen from " $m_{2} \geq(1+y / p)$ " that it can be ensured that a sealing element, when designed and worked or installed to make its minimum necessary sealing stress $y$ small to be ignorable relative to fluid pressure $p$, will never have any leakage before the moment when its maximum unsealing actuation force $F_{u}=$ its sealing actuation $F_{s}$ or its leak-free maintenance factor $m_{2}=1$.

If vessels whose nominal pressure $p_{n} \leq 1 \mathrm{MPa}$ can be designed according to $p_{n}=1 \mathrm{MPa}$, the condition for any qualified pressure-tight or self-energized seal to have no leakage till vessel's burst pressure $\left(p_{b}\right)$ equal to four times the nominal pressure $\left(p_{n}\right)$ is:

- that its maintenance factor $m_{2}=\left(1+y / p_{b}\right)=(1+0.2 / 4)=$ 1 because its minimum necessary sealing stress $y \leq 0.2$ $\mathrm{MPa}$, and thus

— that its minimum total fastener load $F_{\Sigma}=m_{2} F_{u}+F_{i}=A_{u} p_{b}$ $+A_{i} p_{b}=\pi\left(d_{2} / 2\right)^{2} p_{b}=\pi d_{2}^{2} p_{n}$,

where $p_{b}=4 p_{n}=$ vessel's burst pressure, $p_{n}=$ vessel's nominal pressure, $A_{u}=$ sealing contact area, $A_{i}=$ sealing contact minor circle area, $d_{2}=$ sealing contact major circle diameter.

The present standards have mistaken the seal-working or installing stress in position for the sealing stress ${ }^{[13]}$, making a coreless rubber (elastomer) sealing element that is the most difficult to achieve and maintain a fully leak-free connection wrongly regarded as the easiest, and a metallic sealing element that is very easy to achieve and maintain a fully leak-free connection wrongly regarded as the most difficult, and resulting in no design of qualified seals according to the concept of factors $y$ and $m_{2}$.

\section{The Present Standards Have Not Known the Pressure's Sustainability Parameter $p t$, Which Is Equivalent to Not Knowing How to Inspect Seals}


Xu Changxiang, Zhang Xiaozhong, Chen Youjun: It Is Necessary to Abrogate All the International Basic Standards of Seals

As to any pressure vessel or system, it is well-known that the greater its cubage $C$ and leak resistance $R_{L}$, the longer the time $t$ expended on leaking all of its pressure fluid at a constant pressure $p$; the higher the constant pressure $p$, the shorter the time $t$ expended on leaking all of its pressure fluid at the pressure $p$; and vice versa. Hence, it is easy for any ordinary person skilled in the art to think of " $C R_{L}=p t=$ a certain constant".

Pressure's unit $\mathrm{Pa}=\mathrm{N} / \mathrm{m}^{2}=\mathrm{N} \cdot \mathrm{m} / \mathrm{m}^{3}=$ energy stored in a unit cubage of fluid, or the energy $E$ stored in a static pressure vessel or system equals $p C$. Hence, it can be seen from " $p C=p t I_{L} \rightarrow p C / I_{L}=p t \rightarrow C R_{L}=p t$ " that the quantifying value of the pressure-sustaining ability of a vessel or system is the product of pressure $p$ and time $t$ expended on leaking all the fluid therein at a constant pressure $p$ or the product of its two pressure-sustaining parameters, and determined by its intrinsic and unchanged cubage $C$ and leakage resistance $R_{L}$. In other words, " $C R_{L}=p t=$ a certain constant" is really the pressure-sustaining or fluid-leaking law or equation of a pressure vessel or system, and from the equation it can be seen that leakage current $I_{L}=C / t=p / R_{L}$ and leak resistance $R_{L}=p / I_{L}$.

As for any valve:

- the two products $C R_{L}$ and $p t$ of its pressure's sustainability determining and quantifying parameters are identically equal to a certain constant, or $C R_{L}=p t=$ a constant;

- the greater its size, the greater its fluid cubage $C$ too, but

the smaller its leak resistance $R_{L}$, and vice versa;

hence, as for a different size of valves in a similar design, their $C R_{L}=p t \approx$ a similar constant or their $\left(p t / C_{L}\right)<\left(p t / C_{S}\right)$ whether their size is large or small and whether their pressure is high or low, where $C_{L}=$ cubage of large valve and $C_{S}=$ cubage of small valve. Therefore, it always exists that:

- $\Delta t_{L}<\Delta t_{S}$, where $\Delta t_{L}=t / C_{L}=$ time needed for a large valve to leak a drop, and $\Delta t_{S}=t / C_{S}=$ time needed for a small valve to leak a drop, when using a unified test pressure $p$ to test a different size of valves in a similar design, and

- $\Delta p_{L}<\Delta p_{S}$, where $\Delta p_{L}=p / C_{L}=$ pressure decay caused by leaking a drop out of a large valve, and $\Delta p_{S}=p / C_{S}=$ pressure decay caused by leaking a drop out of a small valve, when using a unified test pressure duration $t$ to test a different size of valves in a similar design;

i.e. in order to observe visually detectable leakage on basis of a liquid drop or a gas bubble,

- it is needed to specify a longer test pressure duration for small valves and a shorter test pressure duration for large valves if specifying a unified test pressure $p$ for a different size of valves in a similar design, however the present standards have ignorantly made an opposite specification for test pressure durations ${ }^{[8 \sim 9]}$; and

- no significant leakage test of small valves can be done if specifying a unified test pressure duration $t$ for a different size of valves in a similar design, because leakage will cause a quick test pressure decay and further a quick leakage current decay.

The present standards have not known leakage law or pressure's sustainability of pressure vessels or systems, and so have made an opposite specification for test pressure duration length for inspection of seals, which causes visually detectable leakage to be hidden.

\section{The Present Standards Have Wrongly Speci- fied Different Requirements for Seals ac- cording to Use in Fluid Power Transmitting and Fluid Medium Conveying Systems}

The present standards of seals are respectively set by two different technical committees according to use in fluid power transmitting systems and fluid medium conveying systems. A fluid medium conveying system is aimed at finishing the conveying of fluid medium, and a fluid power transmitting system, at finishing the transmitting of fluid power. However, both need to achieve the effective control of fluid flow and leakage. The two systems have the influence of mechanical vibrations from pumps and environments, and the influence of surge pressures caused by switch of control valves. A fluid medium conveying system needs to be confronted with a higher working temperature, a more dangerous fluid leakage, and greater influences of inner more change in fluid velocity and outer more complex and more violent change in environments of longer and greater pipes than a fluid power transmitting system. Some higher requirements for seals should be specified for use in fluid medium conveying systems than in fluid power transmitting systems, but the present standards have made an opposite specification ${ }^{[8 \sim 10]}$. For example, seals for use in a fluid power transmitting system shall pass, with vessels, an air-tight test at a pressure up to 6.3 $\mathrm{MPa}$, a proof pressure test at a minimum of two times working pressure rating, a burst pressure test at a minimum of four times working pressure rating, a cyclic endurance test at a peak pressure of $133 \%$ of working pressure rating and a vibration test at a pressure of working pressure rating, whereas seals for use in a fluid medium transmitting system are required only to pass an air-tight test at a pressure of 0.6 $\mathrm{MPa}$ and a hydraulic test at a minimum of 1.1 times working pressure rating, but not to pass a proof pressure test with their vessels at a minimum of 1.5 times working pressure rating, although their vessel bodies are also designed to enable them to pass a proof pressure test at a minimum of two times working pressure rating and a burst pressure test at a minimum of four times working pressure rating.

Because the present standards of seals for use in fluid power transmitting systems are set on the base of coreless rubber O-ring seals and, for use in fluid medium conveying systems, on the base of non-rubber-O-ring seals, it can be seen from their respective high and low requirements for seals that the present standards really wrongly regard the coreless rubber O-ring that is the most difficult to achieve and maintain a leak-free connection as the easiest, and, from the specification for the test pressure increase rate limit in fluid power transmitting systems, that coreless rubber O-rings really have influence of lagging of self-energizing deformation. Therefore, it can be said that the present standards of seals are really set on the base of not knowing the science of sealing, and should be thoroughly abrogated. 


\section{Unscientific Flange-Connecting Mechanism and Its Correction of the Present Standards}

Cantilevers (see Fig.5) are a beam securely anchored at only one end to a support, and divided into type $\mathrm{A}$ anchored to a wall (like a horizontal flag pole bolted to a wall), type B created by an extension of simply supported bean (like a springboard), and type $\mathrm{C}$ created by adding a stiff spring to an end board of the beam (like a crane), being of very classic mechanical structures. First, the flange connections of the present standards should be a springboard form of cantilevers (see Fig.6), but cannot be designed and calculated according to a typical cantilever because gaskets cannot provide an enough support for their anchoring end. Second, those flange connections cannot also achieve any fully uniform circumferential compression, which causes the maximum unsealing actuation force $F_{u}$ from calculation to have no significance and thus causes all the designs for seal to be in vain ${ }^{[12]}$. Therefore, the flange connections of the present standards are neither a controllable mechanical structure nor a controllable tight structure.

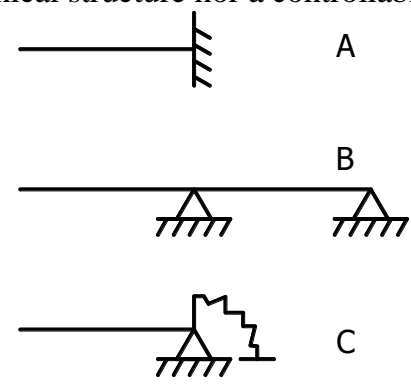

Fig.5 Types of cantilever

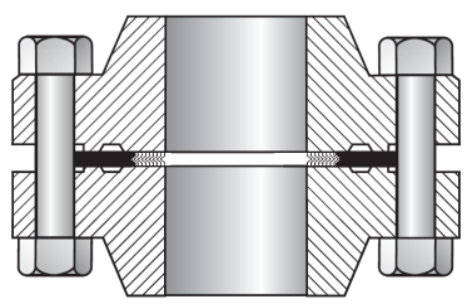

Fig.6 Flange connections specified in the present standards

Xu's flange connection (see Fig.7a) is an ideal solution for correction of the flange connections of the present standards, made up of a fully flat end (top) and a designed end (bottom). On the designed face, besides a macrosawtooth ring (a), there may or may not be microsawtooth rings ( $b_{1}$ and $b_{2}$ ), whose tooth tops are flush with the tooth top of the macrosawtooth ring, and a cavity for O-rings (right) or rectangle rings (left). One, two or three kinds of soft coatings, microsawtooth rings and O-rings or rectangle rings can be optionally used as the sealing element that forms single seal, dual seals or triple seals in one connection.

First, if Xu's flange (see Fig.7a) can be strong enough not to be fastened to bend by fastener force $F$, the flange connection will be a rigid springboard type of cantilevers (see Fig.7b) without any bend after assembled, whose overhanging arm $\mathrm{AB}$ is formed by an extension of a simply supported beam BB', whose lever arm of the first fastened bolt is far shorter than the second fastened bolt opposite the first fastened bolt or whose rise caused by fastening will be effectively constrained and corrected, whose macrosawtooth ring can also ensure that the first round of any uniform tightening effort by fingers cannot cause the flange any rise, and so which cannot have any rise after fully fastened by a sequence of multiple cross-tightening rounds each with an increased uniform torque; i.e. the macrosawtooth ring can result in flanges strong enough having such a very strong anti-rising ability during assembly as to ensure their being parallel joined together.

Second, the strength of the line contact at the top of macrosawtooth ring (a) are macroscopically negligible relative to the strength of the large surface contact between the two flange faces, so that the macrosawtooth ring has no influence on any parallel tight joining of two flanges strong enough.

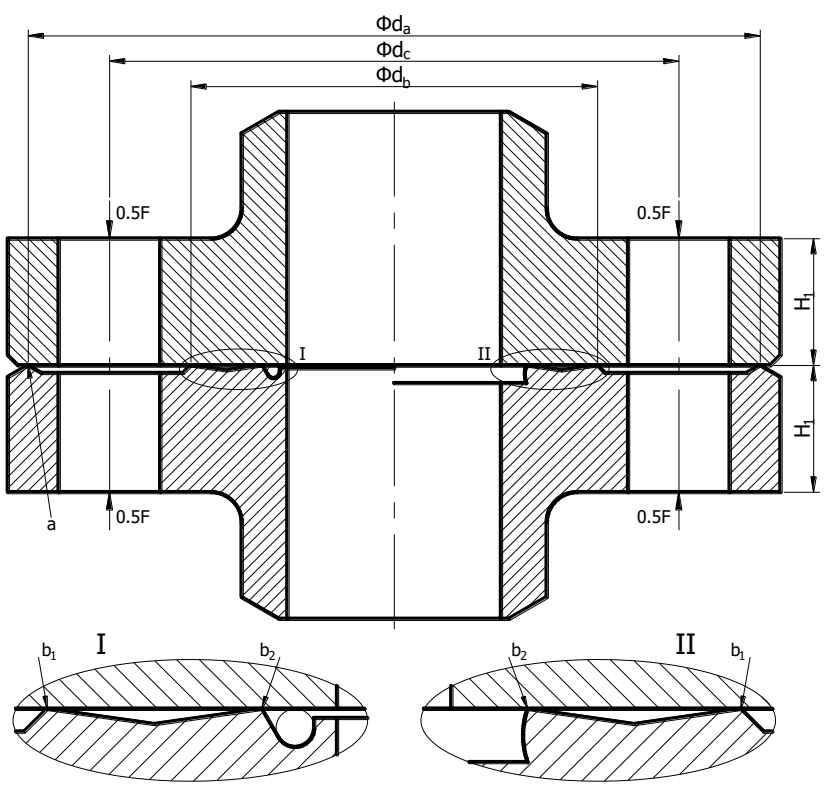

(a)

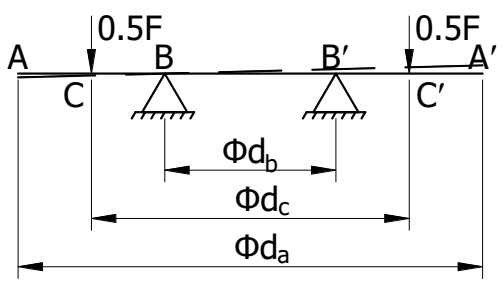

(b)

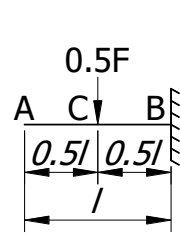

(c)

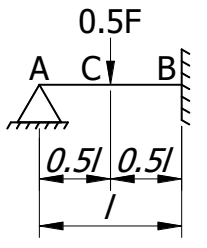

(d)
Fig.7 Xu's flange connection

Third, any cantilever-bending force $F$ that can cause the 
macrosawtooth ring to have a bit of supporting effect on the cantilever will cause the flange connection to become into a beam (Fig.7d) simply supported at one end and anchored at the other end from a cantilever (Fig.7c), causing the bending stress and the reacting stress at support point B to decrease respectively by $62.5 \%$ and $31.25 \%$ and resulting in the bended and collapsed deformation ending and being restored; i.e. the macrosawtooth ring can result in flanges strong enough having a very strong anti-bending and anti-collapsing ability in assembly, ensuring the connection designed according to cantilevers is an ideal cantilever.

Therefore, macrosawtooth rings are undoubtedly no obstacle but an assurance, and also indispensable, for flanges to achieve a uniformly tightened connection. It is due to such that Xu's flange connection designed according to "bending strength of cantilever $\geq$ maximum bending stress caused by maximum total bolt load $F_{\Sigma \max }$ " is both a controllable rigid cantilever structure without tightened bend and a controllable tight structure with a full tightened uniformity.

Because Xu's flange connection can achieve, but the flange connection of the present standards cannot achieve, a fully uniformly tight joining of two flanges, Xu's flange connection separately used soft coatings and microsawtooth rings as a sealing element can be far superior to the flange connection separately used soft and hard seals according to the present standards in both tightness and cheapness, and the reliability of a finalized design of Xu's connections using soft coatings, microsawtooth rings and O-rings or rectangle rings to form their dual and triple seals does not need any leakage inspection. In addition to economic effects resulting from dramatic reduction of its outside diameter, bolt diameter and varieties and sizes, Xu's flange is thoroughly supposed to be substituted for the flanges of the present standards.

\section{Unscientific O-Ring Seal Mechanism and Its Correction of the Present Standards ${ }^{[12]}$}

The O-ring seal of ISO $3601^{[14]}$ is first a design whose working pressure is unknown, then a design whose cavity cannot provide for an O-ring an assembly compression amount that can meet at the same time the requirements of an enough self-energizing exposure and an initial leak-free contact because an assembled O-ring shall be compressed to $r_{a} \geq 0.75 r_{u}$ according to the requirement that its fluid's sealing actuation area shall not be less than its leaking-fluid's unsealing actuation area and also to $r_{a} \leq 0.5 r_{u}$ according to the requirement that it shall be assembled up to its minimum initial tight contact (see Fig.8), and last a design that is the most difficult to achieve a leak-free joint but has been wrongly regarded as the easiest, and hence a design that is wantonly abused and loses control at all.

Xu's circle-based system O-ring seals (see Fig.9) are a round wall cavity of O-ring seals that are based on a polygon's incircle, whose round wall segment has the same center and radius as the incircle in the cross section, whose free O-ring has the same center but not the same diameter as the incircle in the cross section, and hence whose cavity's incircle ring and whose free O-ring have the same nominal cross-sectional circle diameter $d_{s}$ and the same nominal inner diameter $d_{i}$ and outer diameter $d_{o}$, thereby enabling all the global O-ring seals to be unified in one size series, enabling a different structure of O-rings to be used in a different shape of cavities for the same size, and being an ideal solution for correction of the O-ring seals of the present standards.

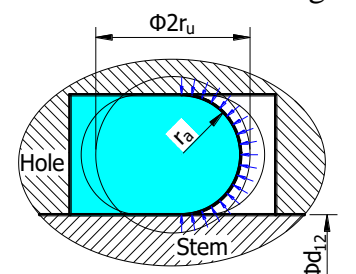

(a) For self-energizing exposure, $r_{a} \geq 0.75 r_{u}$

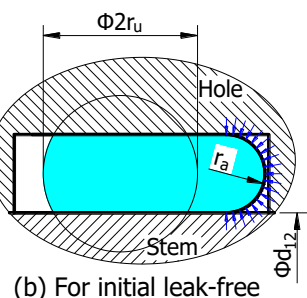
contact, $r_{a} \leq 0.5 r_{u}$

Fig.8 Present $O$-ring seal (b) For initial leak-free (a) O-ring

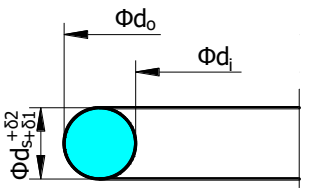

(b) Cavity
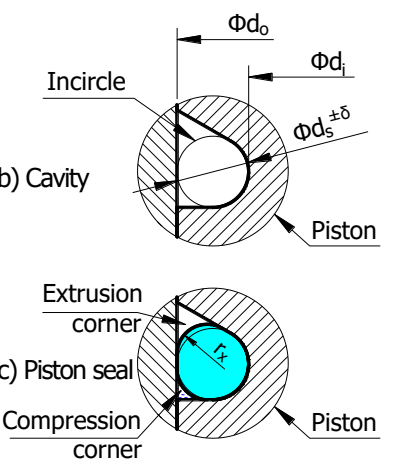

Fig.9 Circle-based system O-ring seal
The cavity of circle-based system O-ring seals has only two corners for their sealing deformation or flow, one being used as their fluid compression corner, and the other, as their free extrusion corner (see Fig.10a). As a fluid pressure arises, - the O-ring will flow from a small space to a great space in its fluid compression corner, which both has no resistance to its sealing deformation or flow and results in a growing fluid actuation area; and

- the O-ring will flow from a great space to a small space in its free extrusion corner, which results in a growing sealing stress.

Hence the circle-based system O-ring has no unnecessary flow and flow resistance that need to consume power, so that a lowest fluid pressure can cause a coreless rubber O-ring to be self-energized up to its fully leak-free state. However, the O-ring of the present standards cannot overcome its huge friction and produce any sealing deformation under a low pressure (see Fig.10b).

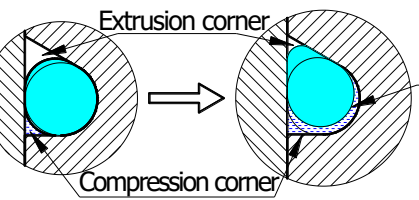

(a) Xu's circle-based O-ring seal

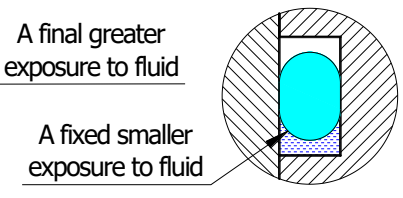

(b) Present seal
Fig.10 Xu's circle-based O-ring seal VS the present seal

The straight wall of cavity of circle-based system O-ring seals is tangential to its round wall, and hence a coreless rubber O-ring will fully leave the round wall momentarily and cause its self-sealing actuation area or power to leap up to its final maximum that can fully meet the requirement of a fully leak-free connection as soon as its radius of flu- 
id-compressed surface in the fluid compression corner grows equal to the radius of the round wall under a pressure (see Fig.10a). However, the O-ring seal of the present standards has only a fixed fluid actuation area for all the time (see Fig.10b).

The cavity's round wall of the circle-based system O-ring seal enables not only a coreless rubber O-ring in time to achieve its full compression from fluid and result in it being up to its fully self-energized state but also a metallic C-ring or O-ring to have a decreased bending moment in its wall and result in it having a greater compression allowance and a greater manufacture tolerance.

The coreless O-ring of the circle-based system O-ring seal in one size can be grouped for its supply or selective use according to its sectional circle diameter or its volume, being capable of both meeting the rigorous requirement for its allowable change in volume and lowering its manufacture cost.

Therefore, all the O-ring seals shall be of a circle-based system.

\section{Unscientific Stem Seal Mechanism and Its Correction of the Present Standards}

The stem cylinder seal of the present standards ${ }^{[15-16]}$ is a pressure-tight packing seal that needs packing to convert a fastener load orthogonally into a sealing load but not possessing any converting condition; i.e. there is no definite converting relation between the fastener load and the sealing load of a stem cylinder seal of the present standards, and it may not be known at all how to provide a safe sealing stress for the seal, causing the seal to lose control at all. The stem shoulder seal of the present standards is a pressure-tight gasket seal. The stem cylinder packing seal and the stem shoul- der gasket seal, if used at the same time, have a common fastener load that can be loaded entirely on the stem shoulder but not entirely on the stem cylinder during installation of the two stem seals, and it is impossible at all to cause them to take effect at the same time. In work of the two stem seals, the fluid pressure on the stem end is to cause the load of the stem shoulder seal to increase but to cause the load of the stem cylinder seal to decrease. Hence, the stem cylinder packing seal and the stem shoulder gasket seal of the present standards are two seals that cannot be coordinated with each other for one stem.

The stem cylinder seal and the stem shoulder seal respectively built with molded truncated equilateral triangle rings 04 (Figs.11 \& 12) and 06 (Fig.12) are loaded via Belleville washers 07 (Fig.11) and 08 (Fig.12), so that an axial fastening force $2 f$ can respectively produce two groups of three identical compressive forces $2 f$ and $2.31 f$ on the three sides of the shoulder-sealing ring 06 and the cylinder-sealing ring 04 all the time, making the relations between the fastening load and the sealing loads clear at glance. Thus the sealing stresses and loads and fastening load for the stem cylinder and shoulder seals can be definitely known from the working pressure of vessels.

Having the outer apex of the equilateral triangle cylinder-sealing ring 04 truncated a bit is to make it have an assembling and wearing allowance. To ensure that each area of two taper surfaces of the cylinder-sealing ring for its being supported and compressed is not smaller than its inner cylinder area or its sealing contact area, the original triangle of the truncated triangle ring 04 shall be the equilateral triangle whose height $h_{c}$ is the side length of another equilateral triangle whose height equals the wall thickness $0.5(D-d)$.

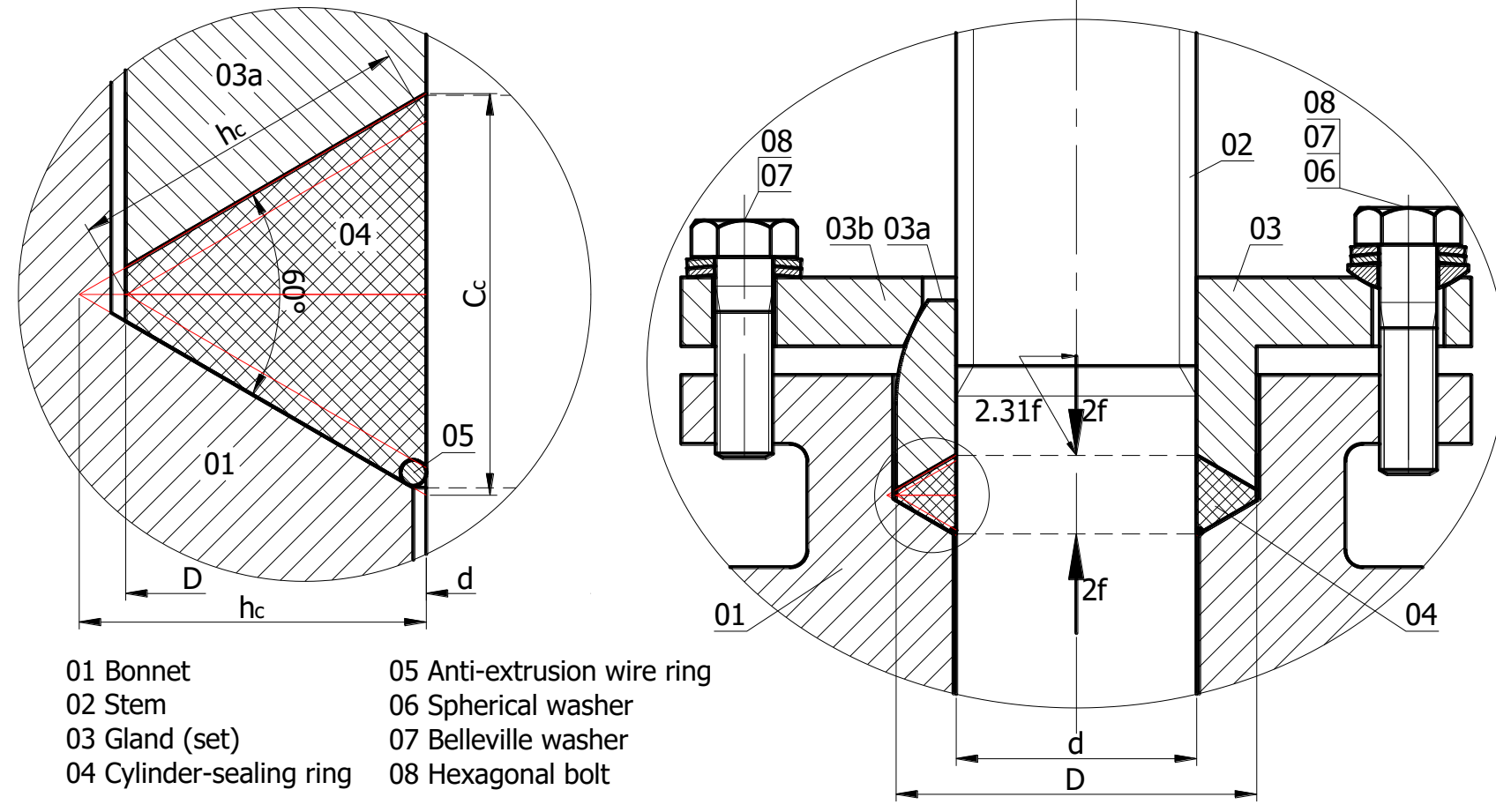

Fig.11 Stem (cylinder) seal for rising stem valves

The orientation of stem 02 is determined with the installa- tion of cylinder-sealing ring 04 into its conical socket in valve 
bonnet or body 01 . In order to ensure that the sealing ring is uniformly compressed respectively in its socket and on its stem, there shall be a clearance small enough between the stem and the central holes of its sealing ring 04 and gland (set) 03, and clearances greater enough between the stem and the hole of its exit in the valve bonnet or body 01 and between the sealing ring 04 with its gland 03 and their containing hole, and the fastener load shall be loaded on the sealing ring 04 via a spherical and conical pair to enable the sealing ring 04 and its gland (set) 03 to swing the stem during installation. In order to prevent the cylinder-sealing ring 04 from being extruded through the clearance between the stem and the bonnet or body at the bottom of the cylinder-sealing ring socket, a soft metallic wire ring 05 shall be added as an anti-extrusion ring.

The outside diameter $D$ of the cylinder-sealing ring and its gland for stems whose outside diameter $d$ is not greater than $50 \mathrm{~mm}$ can be designed according to $D=1.5 d$, and for stems whose outside diameter $d$ is greater than $50 \mathrm{~mm}$, according to $D<1.5 d$. The size and number of bolts 08 (Fig.11) can be determined according to using 4.6 class of bolts to meet the requirement of providing a maximum $80 \mathrm{MPa}$ sealing stress for the stem cylinder, and then their property class higher than 4.6, according to meeting the requirement of providing a maximum sealing stress greater than $80 \mathrm{MPa}$. The material of sealing rings can be selected according to the material tensile strength $\leq$ either 4 or 2 times the working pressure rating. When selected according to the material tensile strength $\leq 2$ times the working pressure rating, the pressure-withstanding limit of the sealing ring is determined by the extrusion strength at the outer top of the sealing ring, and an anti-extrusion core of V-shaped steel strip or mesh shall be added in the sealing ring.

As to a non-rising stem with both a cylinder seal and a shoulder seal, the shoulder seal shall be constructed with a spherical shoulder (see Fig.12). During installation, the spherical shoulder permits the cylinder-sealing ring 04 and its gland 03 to swing the stem 02 to meet the locating requirement of the cylinder-sealing ring socket for the cylinder-sealing ring and the stem and also ensure that the stem shoulder always uses its spherical surface to uniformly press the shoulder-sealing ring 06 and its gland 07, resulting in the cylinder and shoulder seals achieving their uniform being compressed at the same time.

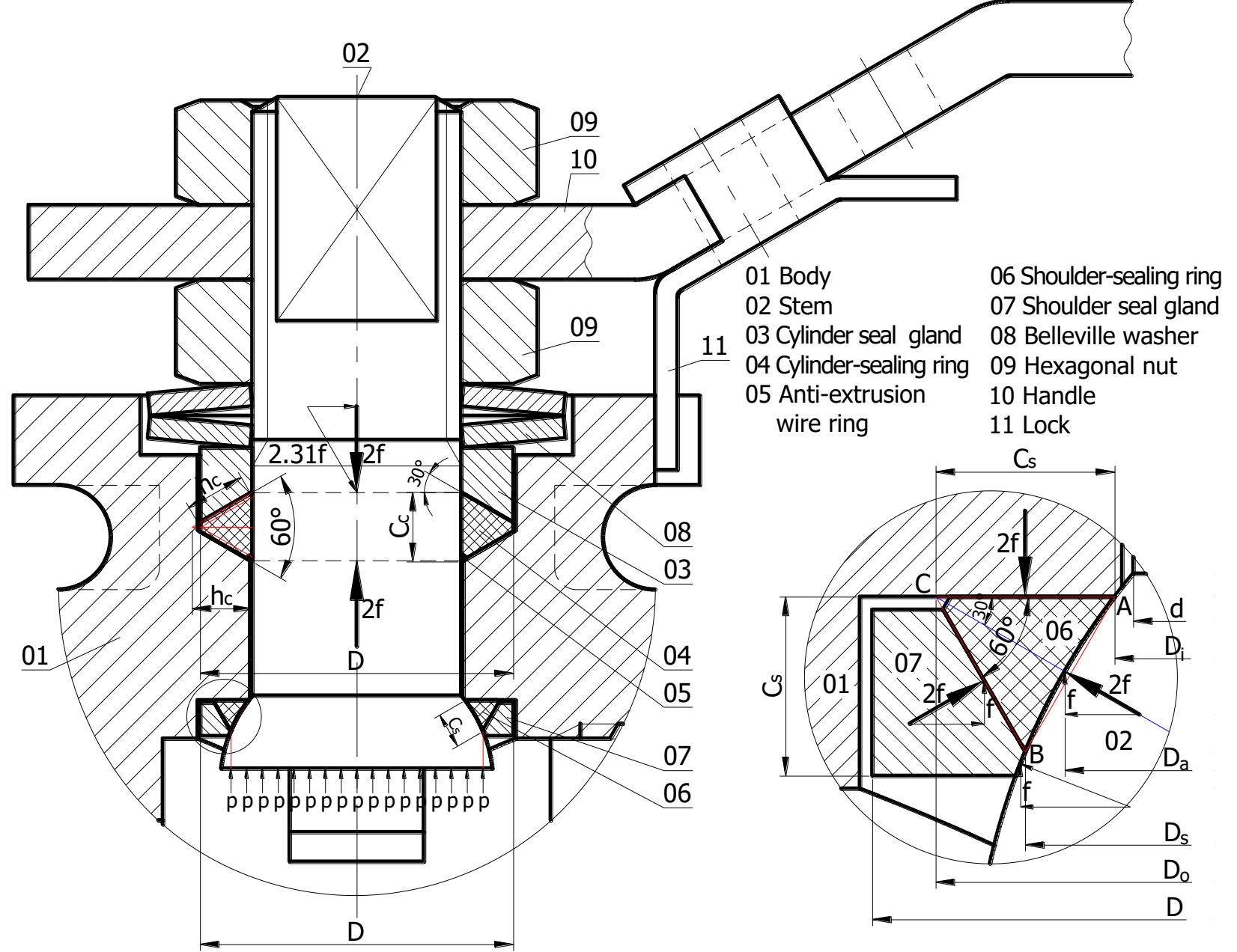

Fig.12 Stem (cylinder and shoulder) seals for ball valves

Between the stem cylinder seal and the stem shoulder seal, also a metallic isolating mating pair made of the spherical shoulder crest and the exit thrust edge of the stem can be arranged (near point A of Fig.12), which is capable of eliminating not only the infinite loading of the stem shoulder seal by tightening force (during installation) and fluid pressure 
(on the stem end) but also the unloading of the stem cylinder seal by fluid pressure (on the stem end), and also capable of resulting in the fluid pressure on the end of gland 07 causing not only an enhanced stem shoulder seal but also such a weakened stem-ejecting-out power as to cause an effect of both lowering the friction of the isolating mating pair and enhancing the stem cylinder seal. Therefore, the triple stem seals made up of an isolating mating pair as well as a stem cylinder seal and a stem shoulder seal that are constructed with triangle rings are the best for a non-rising stem.

When using equilateral triangle rings as the cylinder seal and the shoulder seal of a stem at the same time, it is a common practice in design to have the original triangular side length $C_{c}$ of the cylinder-sealing ring equal to the original triangular side length $C_{s}$ of the shoulder ring. If intending to make the isolating mating pair the third seal of a stem, an increased fastening force $2 f$ will be required to form the third metallic seal during installation, and the design can be practiced according to $C_{c}>C_{s}$ to protect the cylinder seal from being overloaded by the fastening force.

\section{Unscientific Pipe Thread Jointing Mechanism and Its Correction of the Present Standards}

From Xu's sealing theory, it can be known that any pressure-tight joint made on pipe threads shall include an engagement turn of complete jointing threads used to provide a fully (plastically) deformed contact and multiple engagement turns of fastening threads used to provide a fully elastically deformed support for achieving the joint's leak-free state; i.e. a pressure-tight joint made on pipe threads shall not utilize any other mating except for either the mating of internal parallel threads $\mathrm{Rp}$ and external taper threads R of ISO $7{ }^{[17]}$ or the mating of internal straight threads NPS and external taper threads NPT of ASMEB1.20 ${ }^{[18]}$. Besides, the present standards shall specified extra a minimum base diameter $d_{b m i n}$ for external taper pipe threads (see Fig.13) to exclude any possibility that incomplete taper threads come into the joint's tight engagement

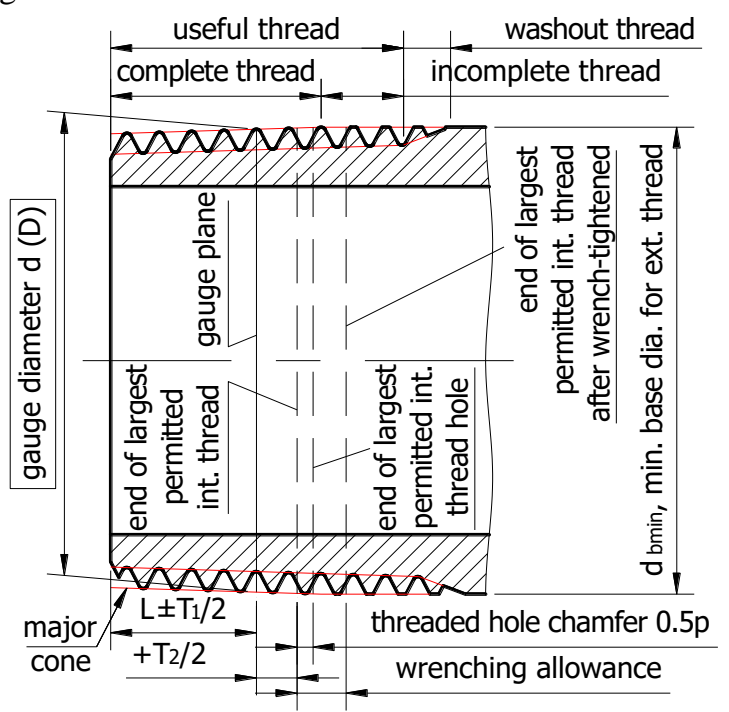

Fig.13 External taper pipe threads $R$ of ISO 7

\section{Conclusions}

Generally speaking, if it cannot be eradicated in designs for leakage to happen in a local long dropping or bubbling way, it cannot be known how much fastener force a seal needs and there will be no effective design of seals because seal's maximum unsealing actuation force, which is equal to the product of fluid pressure and sealing contact area, can only emerge at the moment when a uniform leakage happens. Hence, from the fact that the present standards inspect seals according to "number of liquid drops or gas bubbles leaking to the atmosphere for a unit of time, it has fully been proved that there are no effective design of seals and no qualified seal in the present standardized sealing systems.

Specifically speaking, first, the fastener loads of a flange connection of the present standards are calculated according to its rated working pressure, but its seal-working or installing stress has been wrongly included in its necessary sealing stress, so that a metallic sealing element that is actually very easy to achieve and maintain its fully leak-free connection has been wrongly regarded as the most difficult, causing a fastener load from its seal-designing calculation to have nothing to do with its working pressure. Second, it has been unknown how great working pressure on earth a self-energized O-ring seal specified in the present standards can withstand, and how great fastener load on earth a pressure-tight packing stem seal specified in the present standards is required to achieve its leak-free connection.

As to the test of an identical design of pressure vessels at a fixed pressure, theoretically, a test pressure duration required for a small size of vessels to leak a liquid drop or a gas bubble through their sealing joint is greater than for a great size of vessels, and a test pressure decay caused by leaking a liquid drop or a gas bubble through their sealing joint out of a small size of vessels is greater than out of a great size of vessels; i.e. a small size of pressure vessels need a longer test pressure duration and a more steady test pressure than a great size of vessels in order to be able to have a meaningful test result. However, the present standards not only do not specify the steadiness of test pressure or do not at all consider the influence of leakage-caused pressure decay on the leakage but also make an opposite specification for test pressure duration lengths for leak-free inspection of great and small sizes of vessels, which is undoubtedly equivalent to causing a pressure vessel or system to elude the discovering of visually detectable leakage or to avoid a quality inspection of seals.

All in all, a pressure vessel or system in accordance with the present international standards is at most a product whose fastener load for its tight connection can just achieve no visually detectable leakage for a short time under "a fixed pressure" that causes leakage to get smaller, whose seal's tightness, pressure-withstanding limit and tight sustainability and durability are all unknown, or whose seal's safe reliability is completely unknown. In addition to having no online leakage-monitoring means, any pressure vessel or system in accordance with the present international standards may not work in a leak-free state for a long time, so that a pressure 
vessel or system carrying combustible fluids will explode when meeting the conditions of accumulations of leakage and a fire. Therefore, the liability for vast explosion accidents was/will be almost on the irresistible present international standards of seals but on organizations providing and using a pressure vessel or system. However, it has been so unknown as to cause many persons to be wronged because of accidents, and it is necessary to screen every past accident for a society of equity and justice.

Note: This paper was initially published in Chinese magazine of Petro-Chemical Equipment, 2016, Volume 45 (4): 40-50.

\section{References}

[1] Hydraulic analogy. The Free Encyclopedia, Wikipedia. https://en.wikipedia.org/wiki/Hydraulic_analogy.

[2] Ohm's law. The Free Encyclopedia, Wikipedia. https://en.wikipedia.org/wiki/Ohm\%27s_law\#cite_ref-18

[3] Hagen-Poiseuille equation. The Free Encyclopedia, Wikipedia. http://en.wikipedia.org/wiki/Hagen-Poiseuille_equation

[4] Xu Changxiang, Zhang Xiaozhong, Chen Youjun. Pressure Energy, Resistance and Reactance in Fluid Leak and Flow [J]. Hydraulics Pneumatics \& Seals, 2015, Volume 35 (9): 4-10.

[5] Poiseuille's Law. The Great Soviet Encyclopedia, 3rd Edition. http://encyclopedia2.thefreedictionary.com/Poiseuille's+law

[6] ASTM WK10193 New Recommended Practice for Gasket Constants for Bolted Joint Design [S].
[7] EN 13555 Flanges and Their Joints - Gasket Parameters and Test Procedures Relevant to the Design Rules for Gasketed Circular Flange Connections [S].

[8] ASME B16.34 Valves-Flanged, Threaded, and Welded End [S]

[9] ISO 5208 Industrial Valves - Pressure Testing of Metallic Valves [S].

[10] ISO 19879 Metallic Tube Connections for Fluid Power and General Use - Test Methods for Hydraulic Fluid Power Connections $[\mathrm{S}]$.

[11] Applications Engineering of Garlock. Gasket Constants for the Layman. Garlock's Technical Bulletin, 2004.

[12] Xu Changxiang. Xu's Sealing Theory and Rectangular \& O-Shaped Ring Seals [J]. Petro-Chemical Equipment, 2013, 42(2): 78-85.

[13] ASME Boiler and Pressure Vessel Code, Section VIII, Division 1, Mandatory Appendix 2 Rules for Bolted Flange Connections with Ring Type Gaskets [S].

[14] ISO 3601-2008, Fluid Power System - O-rings [S].

[15] MSS SP-120 Flexible Graphite Packing System for Rising Stem Steel Valves - Design Requirements [S].

[16] MSS SP-132 Compression Packing Systems for Instrument Valves [S].

[17] ISO 7-1-1994 Pipe Threads Where Pressure-Tight Joints Are Made on the Threads - Part 1: Dimensions, Tolerances and Designation [S].

[18] ASME B1.20.1-2013 Pipe Threads, General Purpose [S]. 